\title{
Prevalence and Incidence of Syphilis among Volunteer Blood Donors in Israel
}

\author{
Leibovici Vera, ${ }^{1}$ Donchin Milka, ${ }^{2}$ Strauss-Liviatan Nurith, ${ }^{3}$ and Shinar Eilat ${ }^{4}$ \\ ${ }^{1}$ Department of Dermatology, Hadassah-Hebrew University Hospital, P.O. Box 12018, Jersalem, Israel \\ ${ }^{2}$ Brown School of Public Health, Hadassah University Hospital, Jersalem, Israel \\ ${ }^{3}$ Private Practice, P.O. Box 84027, 90805 Mevaseret Zion, Israel \\ ${ }^{4}$ Magen David Adom National Blood Services, 52621 Ramat Gan, Israel
}

Correspondence should be addressed to Leibovici Vera; vleibovici@hadassah.org.il

Received 26 September 2013; Revised 8 January 2014; Accepted 23 January 2014; Published 22 April 2014

Academic Editor: Rajendra Chaudhary

Copyright (C) 2014 Leibovici Vera et al. This is an open access article distributed under the Creative Commons Attribution License, which permits unrestricted use, distribution, and reproduction in any medium, provided the original work is properly cited.

\begin{abstract}
Data of 1,290,222 volunteer blood donors, in a 5-year period, was analyzed for prevalence and incidence of syphilis. Subsequent testing of donations positive inTreponema pallidum hemagglutination assay included Venereal Disease Research Laboratory and fluorescent Treponemal antibody absorption. Stepwise logistic regression model was used to identify positive syphilis serology. Prevalence of syphilis was $47: 100,000$, similar in men and women and increased significantly with age $(P<0.001)$. Native Israelis had the lowest prevalence rate of syphilis $(21: 100,000)$, while a significantly higher prevalence was found among immigrants from Africa, Eastern Europe, and South America (odds ratios of 19.0, 10.8, and 7.3, resp., $P<0.001$ for each). About 33.2\% of the seropositive donors had evidence of recent infection, and $66.8 \%$ had past infections. Incidence rate reached $8: 100,000$ personyears. Coinfection with HIV, HCV, and HBV was calculated as $8 \%, 1.88 \%$, and $0.37 \%$ for positive donations, respectively. The data support the need to continue screening blood donors in Israel for syphilis and employ preventive measures to populations at risk, in order to improve public health, blood safety, and quality. A subsequent study to assess blood donors' knowledge, attitude, and behavior is planned. In times of global migration this information may be useful to blood services worldwide.
\end{abstract}

\section{Introduction}

Syphilis is a sexually transmitted disease (STD) caused by Treponema pallidum, which can also be transmitted via accidental direct inoculation, transplacenta during pregnancy, and, rarely, via blood transfusion $[1,2]$.

Although the value of routine serologic screening of blood donors for syphilis has been a question in debate for years [3-5], and refrigerated blood components are less infective for syphilis, transmissions through blood components still occur [6]. Therefore, standard operating procedures of blood establishments worldwide include demands/ recommendations for such screening $[7,8]$. New draft guidance for screening, testing, and management of blood donors and components was recently distributed by the FDA [9].

In many parts of the world, the incidence and prevalence of syphilis still remain high in both volunteer and family/replacement blood donors [10-15]. There are numerous reports in high-risk groups in the literature, both from developed and developing countries, indicating rising prevalence and incidence of syphilis [16-20].

In Israel, screening tests for syphilis are conducted in community STD clinics for the general and high-risk populations, by the National Blood Services on each blood unit donated (all collected from volunteer blood donors), and also in all pregnant women. Confirmatory tests for all positive samples are performed exclusively by the Central National Reference Laboratory for Venereal Diseases of the Ministry of Health $(\mathrm{MOH})$, thus forming a national registry for surveillance and followup.

Data reported by the Israeli MOH to the WHO regional office in Europe showed that the incidence of syphilis in the general population in Israel was $2.52,5.8$, and a low 0.7 per 100,000 in 1996, 2001, and 2005, respectively [21]. Although the incidence of syphilis in Israel in the general population in 2005 was rather low, further reports between 2004 and 2009 


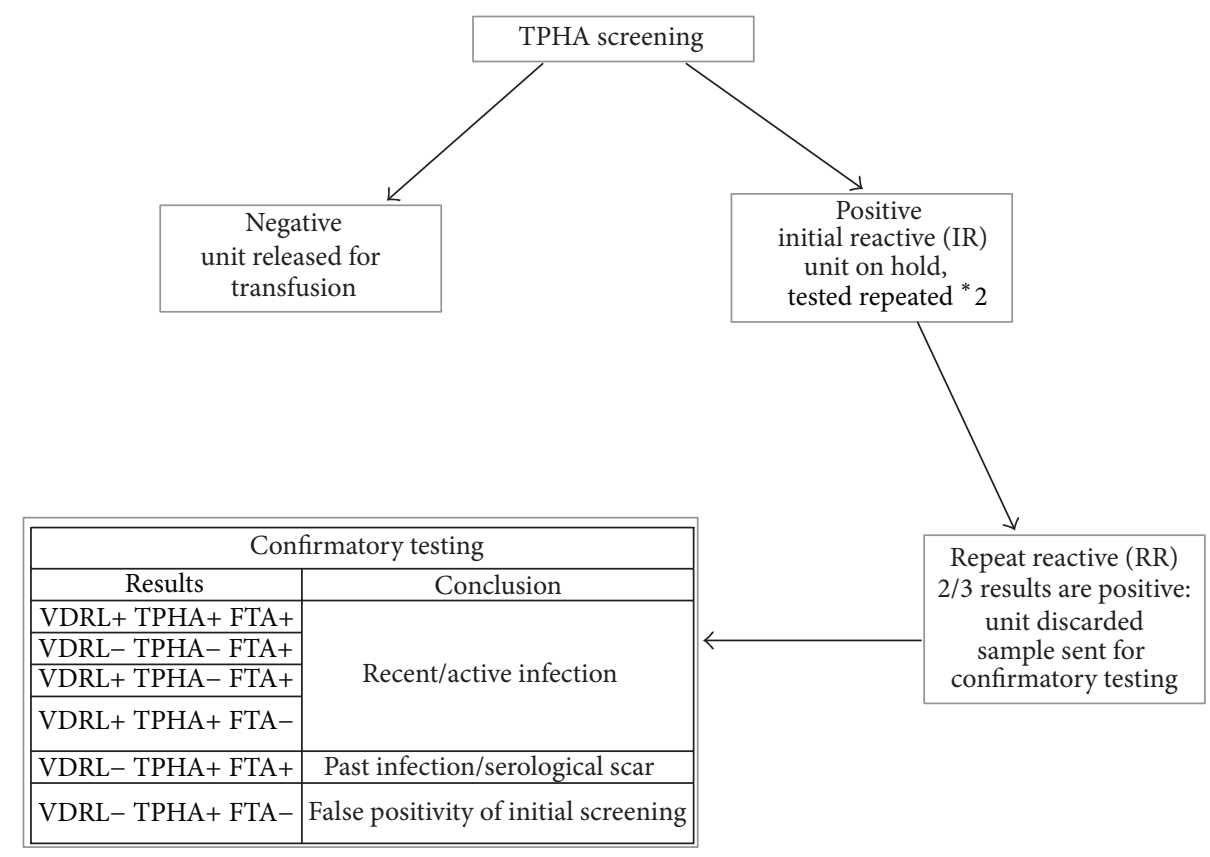

FIGURE 1: Algorithm for syphilis screening and confirmatory testing.

in people with high-risk sexual behavior show an increase of the prevalence and incidence of syphilis in this specific group [22-25]. We studied the prevalence and incidence of syphilis and coinfection with other transfusion-transmitted diseases in volunteer blood donors of the Israeli National Magen David Adom Blood Services (MDABS) during 20052009 to assess the determinants of this sexually transmitted disease in this selected population.

\section{Materials and Methods}

2.1. Study Population. All blood donors in Israel are nonremunerated volunteers. Units are collected throughout the country in mobile vehicles or in fixed-site donor rooms. Before donation each potential donor fills a detailed health history questionnaire, which includes data regarding age, gender, country of birth, and year of immigration (if applicable), and questions concerning the donor's general health, lifestyle and risk behavior, followed by a short private interview, blood pressure and hemoglobin determination, and physical examination. Donors may be disqualified and deferred from donating based on their written and verbal answers or on previous information in the MDABS database. Relevant deferral criteria include, among others, permanent deferral for known HIV or hepatitis, by past testing or selfreport, men who have had sex with men (MSM) since 1977, and people who have been given money for sex (sex workers). A 12-month temporary deferral includes donors who were treated for syphilis or gonorrhea within the previous year and those who had sex with people mentioned above [7]. The study was carried out using the computerized database of MDA Blood Services donors' records for the period 20052009.

\subsection{Laboratory Tests}

2.2.1. Screening for Syphilis (see Figure 1). Tests were done using a specific serological Treponema Pallidum hemagglutination assay (TPHA) of Biokit-Syphagen TPHA autoindirect hemagglutination test (Biokit, Barcelona, Spain), performed on Olympus PK 7200 blood-typing equipment (Olympus, Japan). Each initial reactive sample was further tested in duplicate, according to the manufacturer's instructions. If two out of three results were positive (Repeat Reactive), the unit was discarded and the sample was sent to the National Reference Laboratory for Venereal Diseases of the Israeli Ministry of Health for serological confirmation testing using a different TPHA hemagglutination assay, the nonspecific treponemal assay Venereal Disease Research Laboratory (VDRL), using BD VDRL Antigen (Becton Dickinson, USA), and fluorescent Treponemal absorption test (FTA-ABS) with Trepo-Spot IF, Fluoline $\mathrm{H}$ (Biomerieux France).

According to the confirmatory results donors were defined as

(a) "Recent/Active Infection": if confirmatory results were one of the following: VDRL+ TPHA+ FTA+; VDRL- TPHA- FTA+; VDRL+ TPHA- FTA+ or VDRL+ TPHA+ FTA-, the donors have not been treated and therefore may be infectious [26];

(b) "Past Infection/Serological Scar": if confirmatory results were VDRL- TPHA+ FTA+

The test was defined as false positive when VDRLTPHA + and FTA-.

Prevalence was defined as the number of TPHA positive donors in the total donors' population. Incidence was defined as the number of new TPHA positive donors in the total donors' population. Seroconversion was defined when the 
TABLE 1: Prevalence (\%) of seropositive donors by gender, age, country of birth, and year of immigration.

\begin{tabular}{lccccc}
\hline Variable & Number of donations & Number of donors & Number of seropositives & Prevalence (\%) & 95\% C.I. of prevalence \\
\hline Total & $1,290,222$ & 606,549 & 283 & 0.0467 & $(0.0415,0.0524)$ \\
Gender & & & & & \\
$\quad$ Men & 945,653 & 417,475 & 189 & 0.0453 & $(0.0393,0.0522)$ \\
$\quad$ Women & 344,569 & 189,074 & 94 & 0.0497 & $(0.0406,0.0608)$ \\
Age & & & & & \\
$\quad \leq 24$ & 440,712 & 207,203 & 24 & 0.0116 & $(0.0078,0.0172)$ \\
$25-34$ & 357,888 & 170,781 & 74 & 0.0433 & $0.0345,0.0544)$ \\
$35-44$ & 217,965 & 107,251 & 81 & 0.0755 & $(0.0608,0.0938)$ \\
$\quad \geq 45$ & 273,657 & 121,314 & 104 & 0.0857 & $(0.0708,0.1039)$ \\
Country of origin & & & & & \\
Israel & $1,022,071$ & 474,140 & 101 & 0.0213 & $(0.0175,0.0259)$ \\
$\quad$ Asia & 29,423 & 14,580 & 17 & 0.1166 & $(0.0728,0.1867)$ \\
$\quad$ Africa & 9,921 & 5,047 & 9 & 0.1783 & $(0.0938,0.3386)$ \\
$\quad$ USSR and Eastern Europe & 141,894 & 70,766 & 136 & 0.1922 & $(0.1625,0.2273)$ \\
$\quad$ Western Europe & & & & \\
$\quad$ North America and & 66,106 & 32,370 & 11 & 0.0340 & $(0.0190,0.0608)$ \\
Oceania & & & & \\
$\quad$ South America & 19,159 & 8,863 & 9 & 0.1015 & $(0.0534,0.1929)$ \\
$\quad$ Unknown & 1,648 & 783 & 0 & & \\
Time of immigration & & & & & \\
$\quad$ Israeli born & $1,022,071$ & 474,140 & 101 & 0.0213 & $(0.0175,0.0259)$ \\
$\quad<1990$ & 109,483 & 47,774 & 39 & 0.0816 & $0.0597,0.1116)$ \\
$\quad \geq 1990$ & 142,292 & 72,188 & 136 & 0.1884 & $(0.1593,0.2228)$ \\
$\quad$ Unknown & 16,376 & 12,447 & 7 & 0.0562 & $(0.0272,0.1161)$ \\
\hline
\end{tabular}

current donation from a previously seronegative repeat donor gave a seropositive confirmed reaction.

\subsubsection{Screening for Other Transfusion Transmitted Viruses.} Tests were performed at MDABS laboratories using chemiluminescentimmunoassay for human immunodeficiency virus (HIV), hepatitis B (HBV), hepatitis C (HCV), and human T-lymphotropic virus (HTLV) (Prism, Abbott laboratories, USA), and Individual Unit Nucleic Acid Testing (ID-NAT) for HIV, HBV, and HCV using Procleix Ultrio (Novartis, USA).

2.3. Statistical Analyses. Seroprevalence was defined as the proportion of donors serologically confirmed as positives from the total number of donors and was given with $95 \%$ confidence intervals.

The seroconversion rate per 100,000 person-years was calculated by the number of donors who seroconverted, divided by the total number of person-years between consecutive donations, multiplied by 100,000.

Proportion comparisons were performed with the normal approximation. The chi-square test was used to test the association between categorical variables.

Two models of stepwise logistic regression were used to investigate which variables are associated with total seropositivity (Yes/No) and with recent infection (Yes/No).
We estimated the models with the following explanatory variables: age, country of birth (grouped into Israeli-born, East Europe, and former USSR countries, West Europe, North America, and Oceania, Africa, Asia, and South America), year of immigration (equals 1 for immigration year $\geq 1990$ and 0 before 1990), and the 2 -way interactions between immigration year and country group. Country group was a categorical variable, with Israel as the reference category to each of the other five country groups. We used the forward conditional method for variable selection. The final models are presented. $P$ values are given for the significant variables, and estimates of the odds ratios with their corresponding 95\% confidence intervals are given.

\section{Results}

Data for analysis included a total of 1,290,222 valid blood donations, collected from 605,549 donors, with an average of 2.1 units/donor (range 1-57). About $69 \%$ of the donors were men and $31 \%$ were women. Median age was 29, ranging from 17 to 70 years. Mean age of the donors was $33 \pm 12.4$ years. About 79\% of the blood donors were born in Israel, and 21\% had emigrated from different countries (Table 1).

Out of the 605,549 volunteers who donated blood in 2005-2009, 283 were seropositive, indicating a prevalence of 47 per 100,000. Prevalence rates of all the syphilis seropositive 
TABLE 2: Distribution of donors with recent versus past syphilis by country of origin, age, and time of immigration.

\begin{tabular}{|c|c|c|c|}
\hline & Active infection & Past infection & Total \\
\hline \multicolumn{4}{|l|}{ Country of origin } \\
\hline Total & $94(100 \%)$ & $189(100 \%)$ & $283(100 \%)$ \\
\hline Israel & $36(38.3 \%)$ & $65(34.4 \%)$ & $101(35.7 \%)$ \\
\hline Asia & $8(8.5 \%)$ & $9(4.8 \%)$ & $17(6.0 \%)$ \\
\hline Africa & $4(4.3 \%)$ & $5(2.6 \%)$ & $9(3.2 \%)$ \\
\hline USSR and Eastern Europe & $39(41.5 \%)$ & $97(51.3 \%)$ & $136(48.1 \%)$ \\
\hline $\begin{array}{l}\text { Western Europe } \\
\text { North America and Oceania }\end{array}$ & $4(4.3 \%)$ & $7(3.7 \%)$ & $11(3.9 \%)$ \\
\hline South America & $3(3.2 \%)$ & $6(3.2 \%)$ & $9(3.2 \%)$ \\
\hline \multicolumn{4}{|l|}{ Age (years) } \\
\hline$\leq 24$ & $9(9.6 \%)$ & $15(7.9 \%)$ & $24(8.5 \%)$ \\
\hline $25-34$ & $23(24.5 \%)$ & $51(27.0 \%)$ & $74(26.1 \%)$ \\
\hline $35-44$ & $28(29.8 \%)$ & $53(28.0 \%)$ & $81(28.6 \%)$ \\
\hline$\geq 45$ & $34(36.2 \%)$ & $70(37.0 \%)$ & $104(36.7 \%)$ \\
\hline \multicolumn{4}{|l|}{ Year of immigration } \\
\hline$<1990$ & $15(16.1 \%)$ & $24(13.1 \%)$ & $39(14.1 \%)$ \\
\hline$\geq 1990$ & $42(45.2 \%)$ & $94(51.4 \%)$ & $136(49.3 \%)$ \\
\hline Israeli born & $36(38.7 \%)$ & $65(35.5 \%)$ & $101(36.6 \%)$ \\
\hline
\end{tabular}

donors (with both recent and past infection) analyzed by gender, age, country of origin, and time of immigration to Israel are depicted in Table 1.

Men and women had similar prevalence rates of syphilis of 45 and 49 per 100,000, respectively. There was a significant increase $(P<0.001)$ in the prevalence rate of syphilis with age: donors age 45 years and older, who donated about one-fifth of the total number of units, comprised $36.7 \%$ of the seropositives. Prevalence rates were 7.4, 6.5, and 3.7 times higher in donors age 45 or older, 35-44, and 25-35, respectively, compared to $\leq 24$ years.

When analyzed by country of origin, the highest rates of seropositivity were detected in donors born in Eastern Europe and Africa (192 per 100,000 and 178 per 100,000, resp.), when compared to native Israeli.

Ninety-four of the 283 seropositive donors (33\%) were diagnosed as Recent Infections by the National Reference Laboratory for Venereal Diseases, and 189/283 (67\%) were defined as Past Infection/Serological Scars. The distribution of seropositives between donors defined as recent or past infections was similar between the two groups (Table 2) including following a review of the donors' history and questionnaires.

The logistic regression model (Table 3 ) enables identification of the marginal contribution of the above determinants to the risk of seropositivity, both total and recent infection.

Age increased the risk of syphilis in the given population of blood donors (controlling for the other variables in the model). Every additional year of age adds $6 \%$ to the risk of either total seropositivity or recent infection (odds ratio = $1.06, P<0.001$ ). The interaction of immigration year (above and below 1990) and country of birth group was also significant. More specifically, new immigrants (immigration year $\geq 1990$ ) from Africa, Eastern Europe, and South America had a significantly higher risk for syphilis (with odds ratios of 19.0, 10.8 , and 7.3, resp., $P<0.001$ for each) compared to the risk of Israeli-born donors and those who immigrated before 1990 . Similar results are presented for the recent infection model.

Seroconversion was found in 51 donors, giving an incidence rate of 8 per 100,000 person-years. No significant differences were found when compared to the other seropositive donors, by the studied parameters.

Incidence by year is given in Figure 2.

Coinfection of seropositivity for syphilis with other transfusion-transmitted diseases was found: two out of 25 HIV-positive donors were seropositive for syphilis (8\%), compared to $0.046 \%$ among the HIV-negative blood donors $(P<0.001)$. In addition, $0.37 \%$ and $1.88 \%$ of the seropositive donors for hepatitis $\mathrm{B}$ and $\mathrm{C}$, respectively, were positive for syphilis, compared with 0.046 and $0.045 \%$ among the negative donors for these two hepatitis viruses $(P<0.001$ for each test).

\section{Discussion}

Previous studies of the prevalence of syphilis in Israel mostly took place among people with high-risk sexual behavior, such as MSM or sex workers. Only in one study, reported in 2005 and conducted in a limited region, a low incidence of 0.7 cases of syphilis in 100,000 people was found [21].

The volunteer blood donors are considered a selective population, since they are prescreened for previous diseases and sexual behavior, both by the detailed questionnaire and during a discrete personal interview before donation. However, since blood donation is performed by all citizens, 


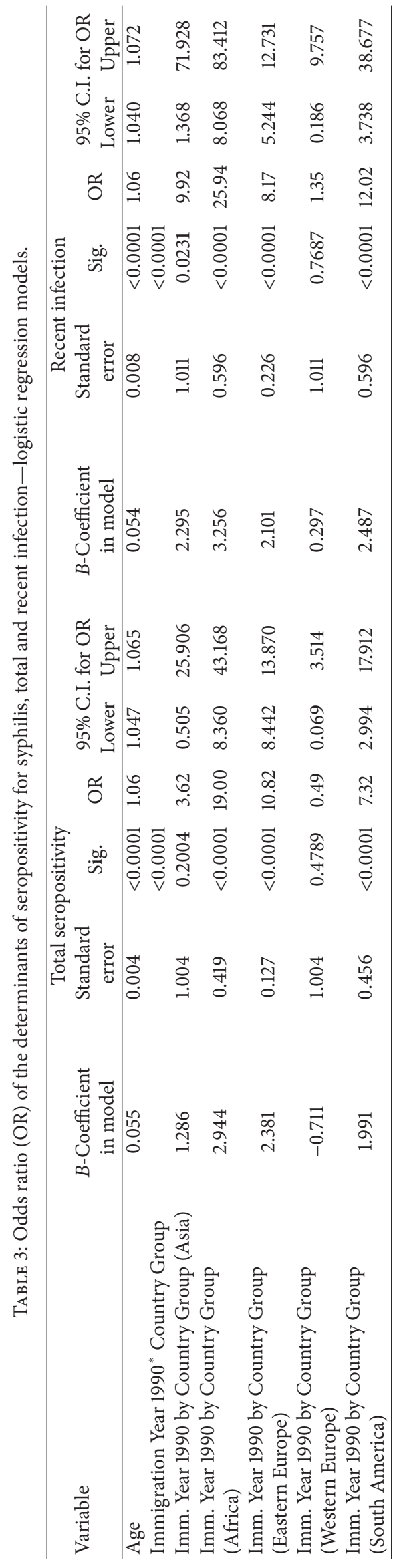




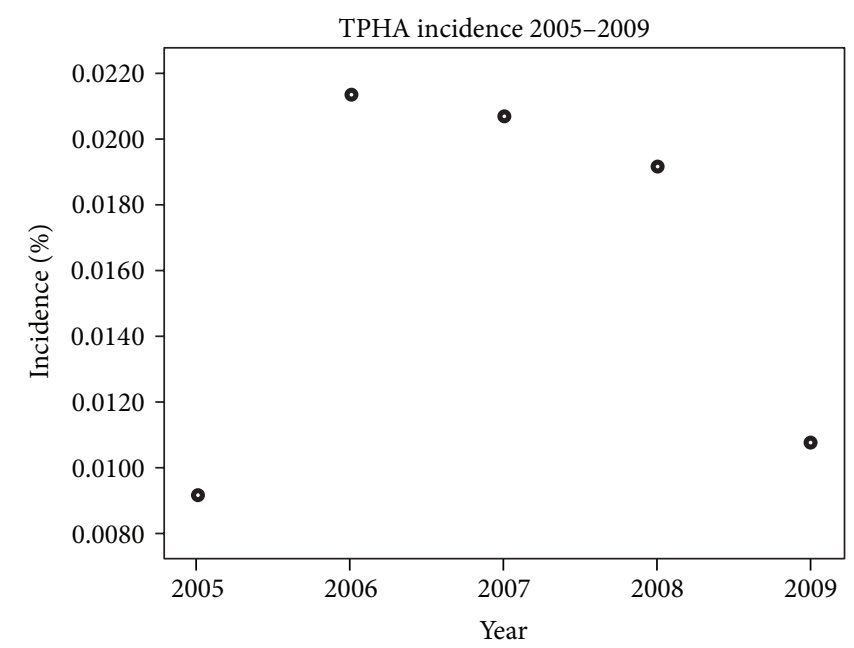

FIGURE 2: Incidence of TPHA seropositivity during the study period.

it could provide an updated picture about incidence and prevalence of syphilis in this population.

Data obtained during the period 2005-2009 show a prevalence of 47 per 100,000, of which 33\% were diagnosed as recently infected and $67 \%$ were defined as past infection/ "serological scars." In addition, an incidence of 8 per 100,000 person-years was detected, which is an 11.4-fold increase since 2005.

Three major variables in our study had statistically significant impact on the risk of seropositivity (either for the entire group or for recent infection): age of the donors, their country of origin, and the time of immigration to Israel.

Age. The prevalence rates of syphilis increased with age. Donors. The 35-44 year age group and those older than 45 years had a 6.5-fold and 7.4-fold higher prevalence of syphilis, respectively, when compared to the younger donors (aged 24 years or less). This is in agreement with others with similar findings among blood donors (18) or who described an increase of primary and secondary syphilis in older adults in the USA [27, 28].

Country of Origin and Oime of Immigration. Israel is a country that absorbs Jewish immigrants from all over the world. In particular, from 1990 to 2001 more than 900,000 immigrants came to the country (13\% of the total Israeli population), mostly from the former Soviet Union and from Africa [29].

Our study revealed that the new immigrants from Africa and from Eastern Europe who arrived in Israel after 1990 had a 19-fold and 10.8-fold (resp.) higher risk for syphilis seropositivity than donors born in Israel. The findings were similar to the prevalence in their countries of origin, as reported by the Israeli MOH [30] and the 2001 WHO European Health database [21]. These results are in accordance with the high incidence of syphilis in the former communist countries of Eastern Europe, with rates of 262, 245, and 150 cases per 100,000 in the Russian Federation, Kazakhstan and Ukraine, respectively [21], and with data published from Africa in 2001, with infection rates of 1000, 300-400, and 100-200 cases per 100,000 found in Zambia, Kenya, and Benin, respectively [31].
The study also depicted an association between syphilis and other transfusion-transmitted diseases such as HIV, hepatitis $\mathrm{B}$, and $\mathrm{C}$, when compared to the donor population that was seronegative for syphilis, and it concurred with our previous observations of higher prevalence of hepatitis $C$ among blood donors who immigrated to Israel from the former USSR [32].

It is worth mentioning that about $62 \%$ of the units were donated by native Israelis, with the lowest risk for recent infection, thus contributing to the safety of the national inventory.

In view of the ongoing discussion regarding the recent FDA's draft Recommendation for Screening, Testing and Management of Blood Donors, and Blood Components Based on Screening Tests for Syphilis [9], our findings demonstrate that screening for syphilis may still retain certain value in Israel and should be considered in some other regions in the world, depending on their blood donors' epidemiology data.

Given the high prevalence of syphilis in Israel allied with active immigration, special attention should be invested in targeting preventive measures to the blood donor populations and populations at risk. A future study is planned to assess the knowledge, attitude, and behavior of the volunteer blood donors' population. These measures will most probably improve public health and increase blood safety and quality.

In times of global migration the reported information may be useful to blood services worldwide.

\section{Conflict of Interests}

The authors declare that there is no conflict of interests regarding the publication of this paper.

\section{Acknowledgments}

The authors express our gratitude to Mrs. Becky Rubin for preparing the data set and to Dr. Veronica Gendelman, from MDA Blood Services. 


\section{References}

[1] E. Van Dyck, R. Musonda, L. Zekeng et al., "Study Group on Heterogeneity of HIV Epidemics in Zeltser R, Kurban AK. Syphilis," Clinics in Dermatology, vol. 22, pp. 461-468, 2004.

[2] C. Gardella, A. A. Marfin, R. H. Kahn, E. Swint, and L. E. Markowitz, "Persons with early syphilis identified through blood or plasma donation screening in the United States," Journal of Infectious Diseases, vol. 185, no. 4, pp. 545-549, 2002.

[3] J. Case, "Serologic test for syphilis: a discredited surrogate test for HIV infection," Transfusion, vol. 38, no. 2, p. 218, 1998.

[4] S. Orton, "Syphilis and blood donors: what we know, what we do not know, and what we need to know," Transfusion Medicine Reviews, vol. 15, no. 4, pp. 282-291, 2001.

[5] L. M. Katz, "A test that won't die: the serologic test for syphilis," Transfusion, vol. 49, no. 4, pp. 617-619, 2009.

[6] S. Attaullah, S. Khan, and J. Khan, "Trend of transfusion transmitted infections frequency in blood donors: provide a road map for its prevention and control," Journal of Translational Medicine, vol. 10, no. 1, article 20, 2012.

[7] Standards for Blood Banks and Transfusion Services, AABB, 28th edition, 2012.

[8] Guide for the Preparation: Recommendation for Screening, Testing and Management of Blood Donors and Blood Components Based on Screening Tests for Syphilis, European Directorate for the Quality of Medicines and Healthcare, 16th edition, 2011.

[9] Guidance for the Industry: Recommendation for Screening, Testing and Management of Blood Donors and Blood Components Based on Screening Tests For Syphilis, FDA, CBER, 2013.

[10] M. L. K. Mogtomo, S. L. Fomekong, H. F. Kuate, and A. N. Ngane, "Screening of infectious microorganisms in blood banks in Douala (1995-2004)," Cahiers Sante, vol. 19, no. 1, pp. 1-8, 2009.

[11] S. Zou, E. P. Notari, C. T. Fang, S. L. Stramer, and R. Y. Dodd, "Current value of serologic test for syphilis as a surrogate marker for blood-borne viral infections among blood donors in the United States," Transfusion, vol. 49, no. 4, pp. 655-661, 2009.

[12] J. Liu, Y. Huang, J. Wang et al., "The increasing prevalence of serologic markers for syphilis among Chinese blood donors in 2008 through 2010 during a syphilis epidemic," Transfusion, vol. 52, pp. 1741-1749, 2012.

[13] Z. H. Ji, C. Y. Li, Y. G. Lv et al., "The prevalence and trends of transfusion-transmissible infectious pathogens among firsttime, voluntary blood donors in Xi'an, China between 1999 and 2009," International Journal of Infectious Diseases, vol. 17, pp. 259-262, 2013.

[14] J. J. Noubiap, W. Y. Joko, J. R. Nansseu, U. G. Tene, and C. Siaka, "Sero-epidemiology of human immunodeficiency virus, hepatitis $\mathrm{B}$ and $\mathrm{C}$ viruses, and syphilis infections among firsttime blood donors in Edéa, Cameroon," International Journal of Infectious Diseases, vol. 17, no. 10, pp. e832-e837, 2013.

[15] S. L. Gottlieb, V. Pope, M. R. Sternberg et al., "Prevalence of syphilis seroreactivity in the United States: Data from the National Health and Nutrition Examination Surveys (NHANES) 2001-2004," Sexually Transmitted Diseases, vol. 35, no. 5, pp. 507-511, 2008.

[16] Department of Health and Human Services Cf DCaP National Center For HIV, STD and TB Prevention, Division of STD Prevention, 2005.

[17] P. Pathela, S. L. Braunstein, J. A. Schillinger, C. Shepard, M. Sweeney, and S. Blank, "Men who have sex with men have a 140fold higher risk for newly diagnosed HIV and syphilis compared with heterosexual men in New York City," Journal of Acquired Immune Deficiency Syndromes, vol. 58, no. 4, pp. 408-416, 2011.

[18] K. Salado-Rasmussen, T. L. Katzenstein, J. Gerstoft et al., "Risk of HIV or second syphilis infection in Danish men with newly acquired syphilis in the period 2000-2010," Sexually Transmitted Infections, vol. 89, no. 5, pp. 372-376.

[19] E. Muldoon and F. Mulcahy, "Syphilis resurgence in Dublin, Ireland," International Journal of STD and AIDS, vol. 22, no. 9, pp. 493-497, 2011.

[20] C. Hao, H. Yan, H. Yang et al., "The incidence of syphilis, HIV and HCV and associated factors in a cohort of men who have sex with men in Nanjing, China," Sexually Transmitted Infections, vol. 87, no. 3, pp. 199-201, 2011.

[21] WHO Meeting on Prospects For the Public Health Approach To the Prevention and Care of Sexually Transmitted Infections in Countries of Eastern Europe and Central Asia, WHO European Health for All Database, 2001.

[22] E. Hefer, L. Rubin, I. Volovik, and S. Rishpon, "Sexually transmitted disease morbidity trends in the Haifa District during 2001-2004," Harefuah, vol. 146, no. 6, pp. 425-428, 2007.

[23] H. Joffe, E. Bamberger, S. Nurkin et al., "Sexually transmitted diseases amon patients with human immunodeficiency virus in Northern Israel," Israel Medical Association Journal, vol. 8, no. 5, pp. 333-336, 2006.

[24] Y. Linhart, T. Shohat, Z. Amitai et al., "Sexually transmitted infections among brothel-based sex workers in Tel-Aviv area, Israel: high prevalence of pharyngeal gonorrhoea," International Journal of STD and AIDS, vol. 19, no. 10, pp. 656-659, 2008.

[25] T. Brosh-Nissimov, Z. Mor, E. Avramovich et al., "Syphilis outbreak among men who have sex with men, Tel Aviv, Israel, 2008-2009," Israel Medical Association Journal, vol. 14, no. 3, pp. 152-156, 2012.

[26] S. Nesteroff, RCPA Serology, QAP, Serology, 2004.

[27] L. A. Jeffers and M. C. DiBartolo, "Raising health care provider awareness of sexually transmitted disease in patients over age 50," Medsurg Nursing, vol. 20, no. 6, pp. 285-290, 2011.

[28] J. Holden and L. Trachtman, "Increase in primary and secondary syphilis cases in older adults in Louisiana," The Journal of the Louisiana State Medical Society, vol. 163, no. 6, pp. 308-311, 2011.

[29] The Israeli Central Bureau of Statistics, Immigrant Population From the Former USSR, Demographic Trends, 2001.

[30] Notifiable Infectious Diseases in Israel- 60 Years of Surveillance (1951-2010), Center For Disease Control (ICDC) and the Division of Epidemiology, Public Health Services, The Israeli MOH, 2012.

[31] A. Buvé, H. A. Weiss, M. Laga et al., "The epidemiology of gonorrhoea, chlamydial infection and syphilis in four African cities," AIDS, vol. 15, no. 4, pp. S79-S88, 2001.

[32] H. Kerzman, M. S. Green, and E. Shinar, "Risk factors for hepatitis $\mathrm{C}$ virus infection among blood donors in Israel: a casecontrol study between native Israelis and immigrants from the former Soviet Union," Transfusion, vol. 47, no. 7, pp. 1189-1196, 2007. 


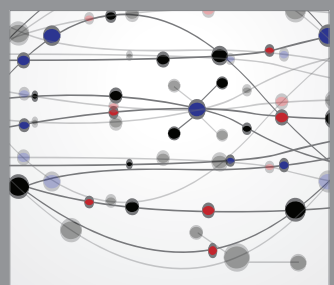

The Scientific World Journal
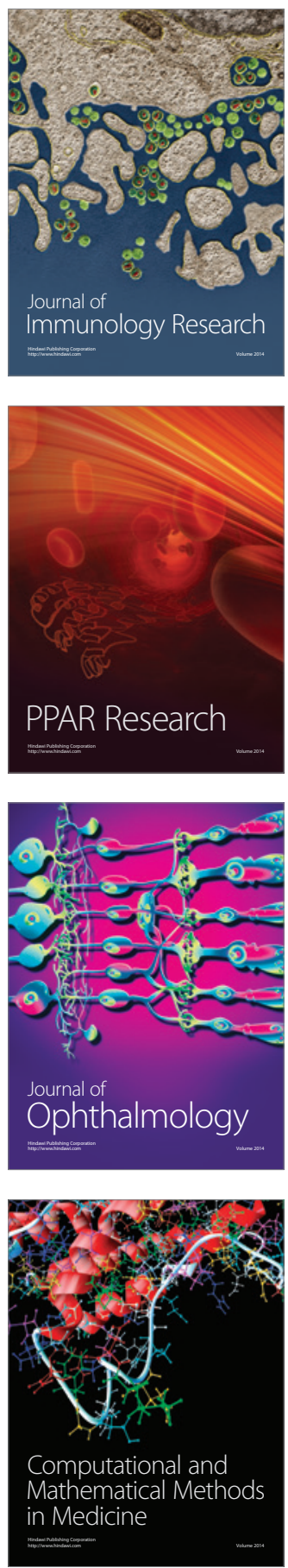

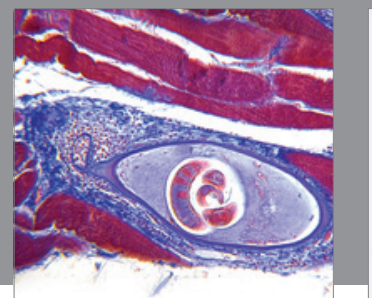

Gastroenterology

Research and Practice
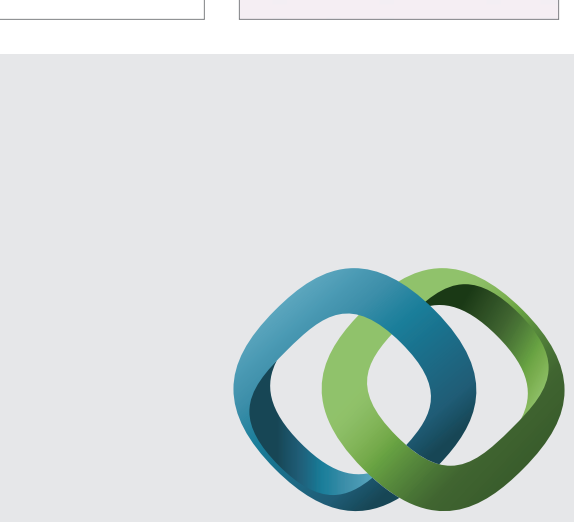

\section{Hindawi}

Submit your manuscripts at

http://www.hindawi.com
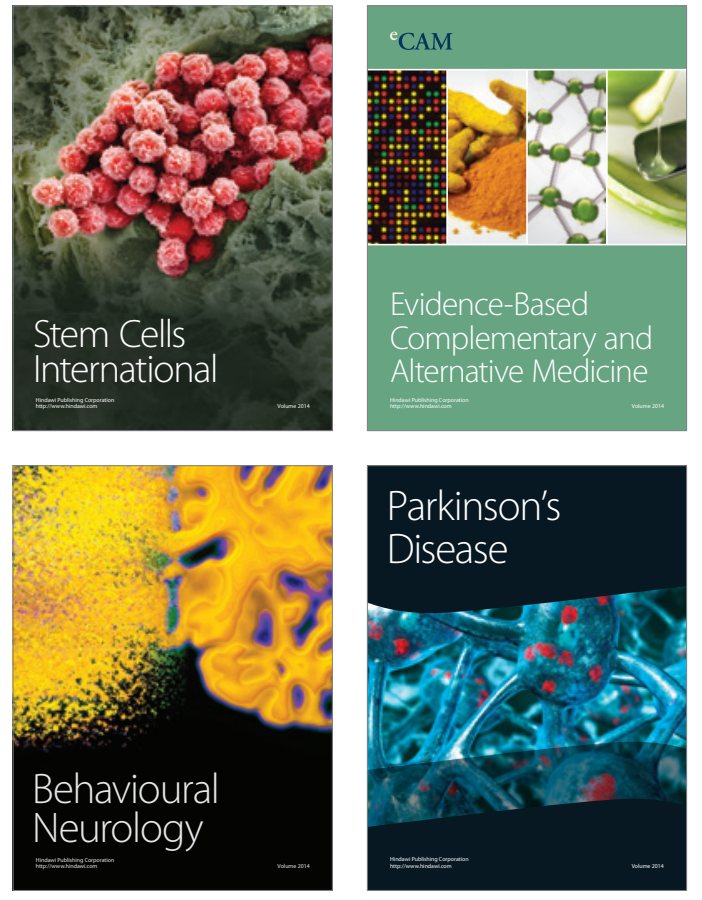
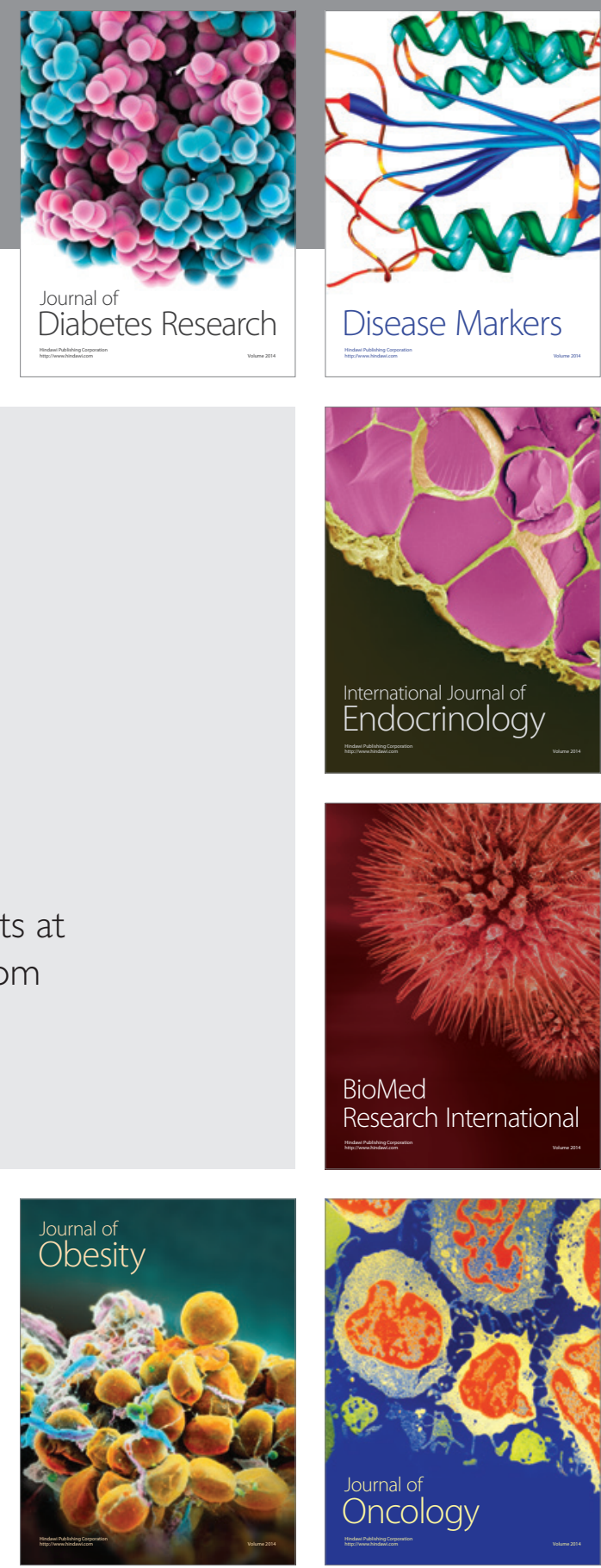

Disease Markers
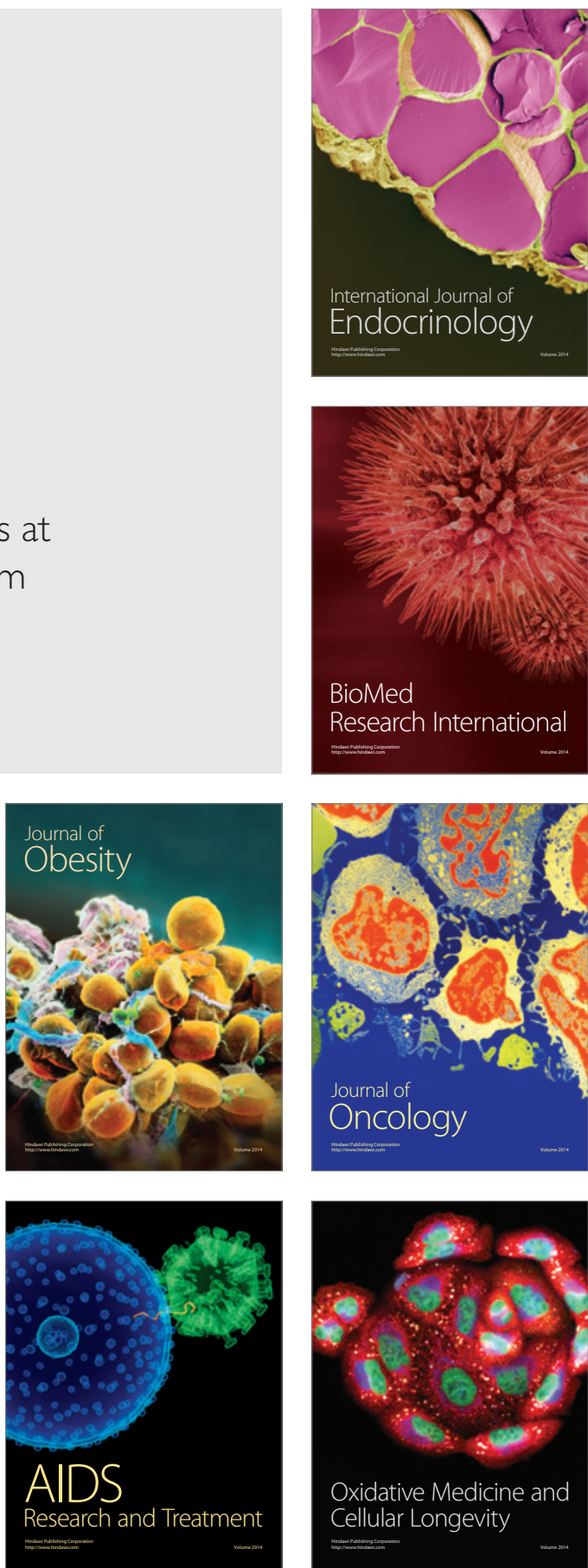